\title{
Propagation d'ondes ultrasoniques dans les roches anisotropes
}

\author{
Ultrasonic waves propagation in anisotropic rocks
}

\author{
P. CUXAC, F. HOMAND \\ Laboratoire de Géomécanique, ENSG*
}

Rev. Franç. Géotech. n 59, pp. 49-55 (avril 1992)

\section{Résumé}

Cet article présente des résultats sur les mesures par ondes ultrasoniques en milieu anisotrope. Dans un premier temps, l'étude de la propagation d'ondes ultrasoniques permet de déterminer le degré de symétrie et l'influence de divers paramètres (contrainte, saturation...). Dans un deuxième temps, les coefficients élastiques sont calculés. On constate une influence importante de la contrainte et du taux de saturation en eau, suivant une direction orthogonale au plan d'anisotropie. Les modules élastiques sont aisément calculés, et ce, avec une dispersion très réduite. Le principe de la biréfringence des ondes $\mathrm{S}$, rappelé ici, peut permettre d'obtenir plus de renseignements sur l'anisotropie.

\begin{abstract}
This paper presents results from ultraconic waves measures in anisotropic media. First, the study of ultrasonic wave propagation permit the determination of symmetrical degree and the influence of differents parameters. The stiffness matrix are calculated. We establish an important influence of the stress and of the saturation rate in a direction orthogonaly to anisotropic plane. The elastic modulus are easily calculated with a reduced dispersion. The $\mathrm{S}$ wave scattering principle remind here, can permit to obtain more information about the anisotropy.
\end{abstract}

- Rue du Doyen-Marcel-Roubault, 54501 Vandceuvre-lès-Nancy. 


\section{INTRODUCTION}

Selon l'échelle à laquelle on se place, toutes les roches sont anisotropes. Dans les études de mécanique des roches, il est important de pouvoir quantifier les anisotropies, mais également de déterminer leurs directions et leur nature (anisotropie planaire, linéaire...).

L'étude de la célérité d'ondes ultrasoniques permet, de façon rapide, une telle détermination avec une très bonne précision.

Les célérités d'ondes de compression et de cisaillement peuvent ensuite être utilisées pour le calcul de la matrice de raideur du matériau étudié.

\section{PRÉSENTATION DES ANISOTROPIES ÉTUDIÉES}

Nous présenterons des résultats obtenus sur des roches isotropes transverses ou orthotropes de révolution (système hexagonal) et sur des roches orthotropes (système orthorhombique).

Un matériau orthotrope de révolution est schématisé figure $1 \mathrm{a}$ avec la matrice de raideur correspondante. Il peut s'agir de roches possédant un plan de schistosité $\left(\mathrm{S}_{1}\right)$.

La figure $1 \mathrm{~b}$ schématise un matériau orthotrope avec sa matrice de raideur. Il peut s'agir d'une roche à plan

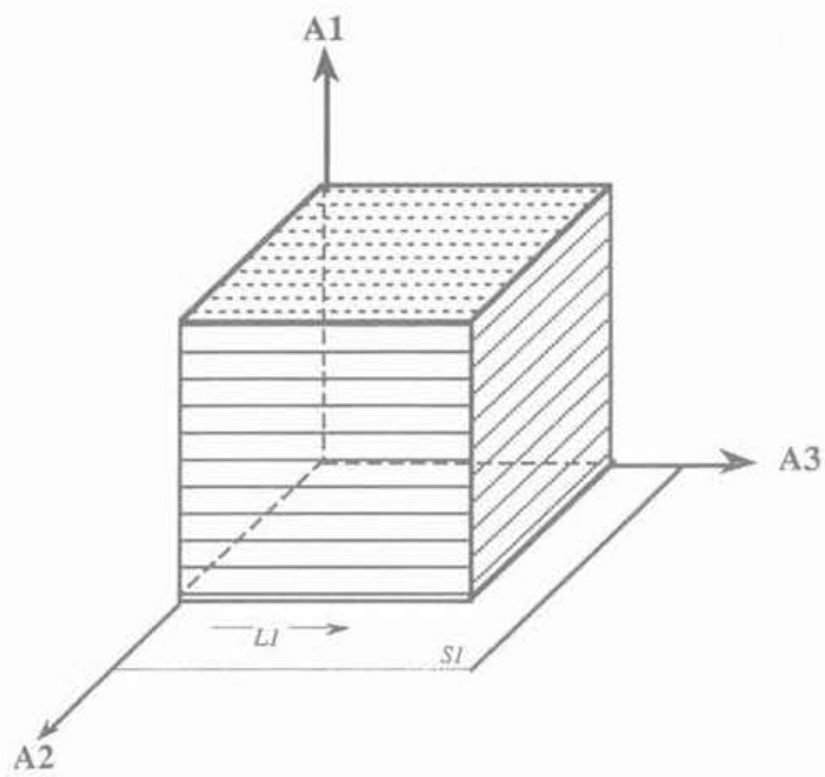

de schistosité ou de foliation $\left(\mathrm{S}_{1}\right)$ contenant une linéation minérale $\left(\mathrm{L}_{1}\right)$. Nous voyons donc que, selon la symétrie considérée, le nombre d'éléments indépendants dans la matrice de raideur sera différent.

On distinguera les roches à anisotropie planaire discontinue (schistes) des roches à anisotropie planaire continue (gneiss).

Afin de déterminer l'anisotropie, il est bon de mesurer les vitesses de propagation d'ondes suivant différentes directions sur un même échantillon. Pour cela nous avons confectionné des polyèdres à 18 ou 26 faces à partir d'échantillons cubiques. Les vitesses mesurées sont ensuite reportées sur un diagramme de WULFF. La connaissance des vitesses d'ondes P et $\mathrm{S}$ dans plusieurs directions permet de calculer la matrice de raideur.

\section{3. ÉTUDE DES VITESSES DE PROPAGATION}

\subsection{Influence de l'anisotropie de structure}

Nous présentons ici les résultats obtenus sur un schiste d'Angers et sur un gneiss de Bretagne (fig. 2a et 2b).

Dans le cas du schiste, on observe une vitesse minimale mesurée perpendiculairement au plan de schis-
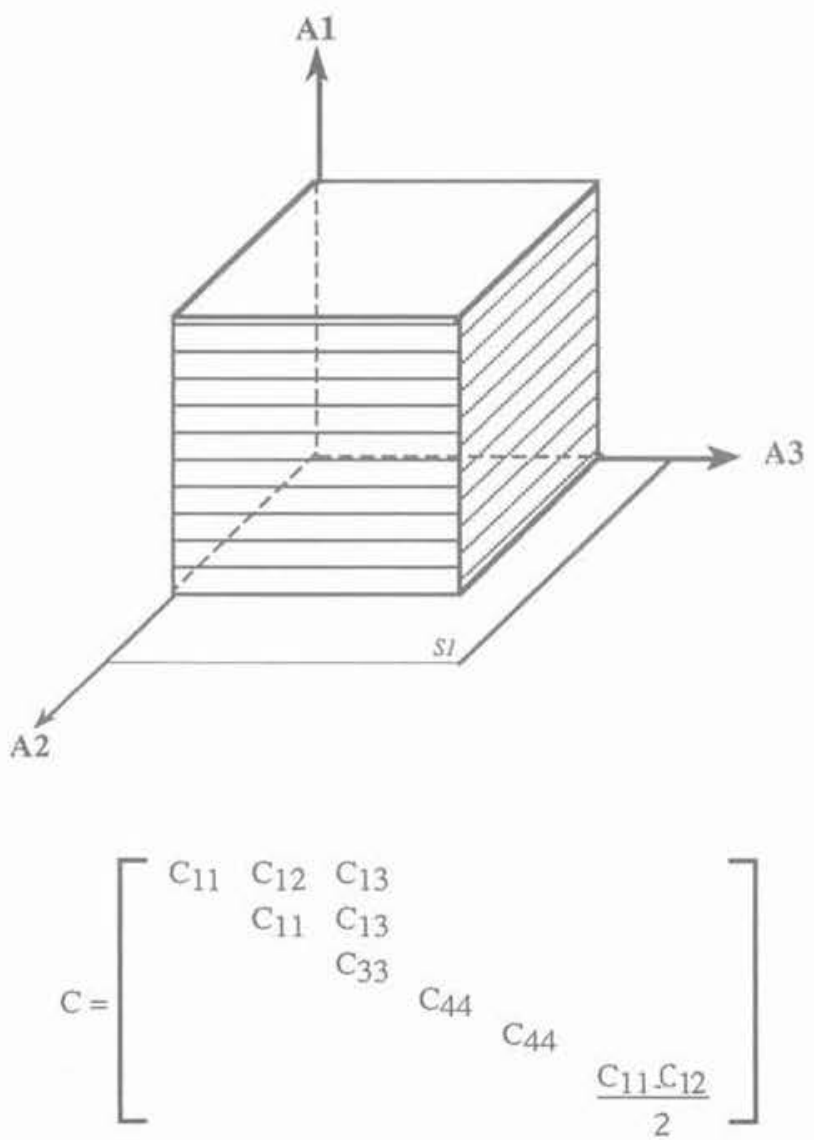

Fig. 1b. - Corps isotrope tranverse.

Fig. 1b. - Transversaly isotropic sold
Fig. 1a. - Corps orthotrope.

Fig. 1a, - Orthotropic solid. 


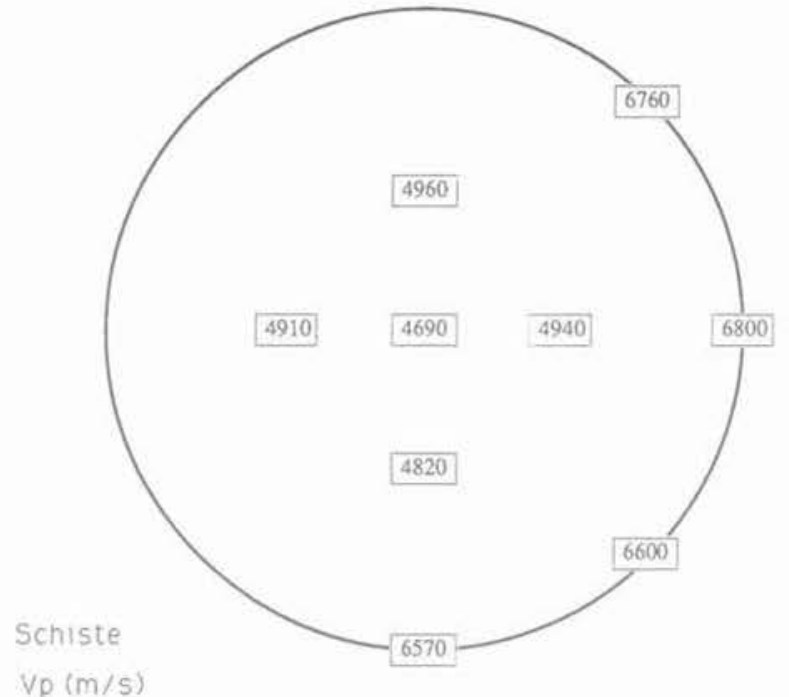

Fig. 2a. - Vitesses d'une onde $P$ dans un schiste.

Fig. $2 a$. - P wave velocity in a slate.

tosité. Dans ce plan, une direction est légèrement plus rapide. On a ici dominance de l'anisotropie planaire.

Dans le cas du gneiss, il apparaît un écart de vitesse important dans le plan de foliation représenté ici par le cercle extérieur du diagramme de WULFF. Pour cette roche, on constate donc une anisotropie linéaire très marquée.

GUYADER et al. (1986) déterminent la présence de deux types d'anisotropie à l'aide des deux paramètres suivants :

- anisotropie majeure :

$$
\Delta \mathrm{M} \%=100\left[1-\frac{2 \mathrm{~V}_{3}}{\mathrm{~V}_{1}+\mathrm{V}_{2}}\right]
$$

- anisotropie mineure:

$$
\Delta \mathrm{m} \%=100\left[\frac{2\left(\mathrm{~V}_{1}-\mathrm{V}_{2}\right)}{\left(\mathrm{V}_{1}+\mathrm{V}_{2}\right)}\right]
$$

avec $V_{1}>V_{2}>V_{3}$

On trouve ainsi, dans le cas du schiste une anisotropie majeure de l'ordre de $30 \%$ et une anisotropie mineure de l'ordre de $5 \%$. Cette dernière anisotropie, due à une linéation minérale n'est décelable qu'à partir d'une étude microscopique minutieuse. La direction et l'intensité de cette anisotropie sont très constantes sur tous les échantillons testés.

Pour le gneiss, l'anisotropie due à la foliation est de $6,5 \%$, alors que la linéation induit une anisotropie de $14 \%$.

\subsection{Influence de la saturation}

La figure 3 donne les vitesses mesurées en immersion sur un polyèdre de schiste saturé.

La très faible porosité de ce matériau explique que les variations de célérité des ondes soient peu impor-

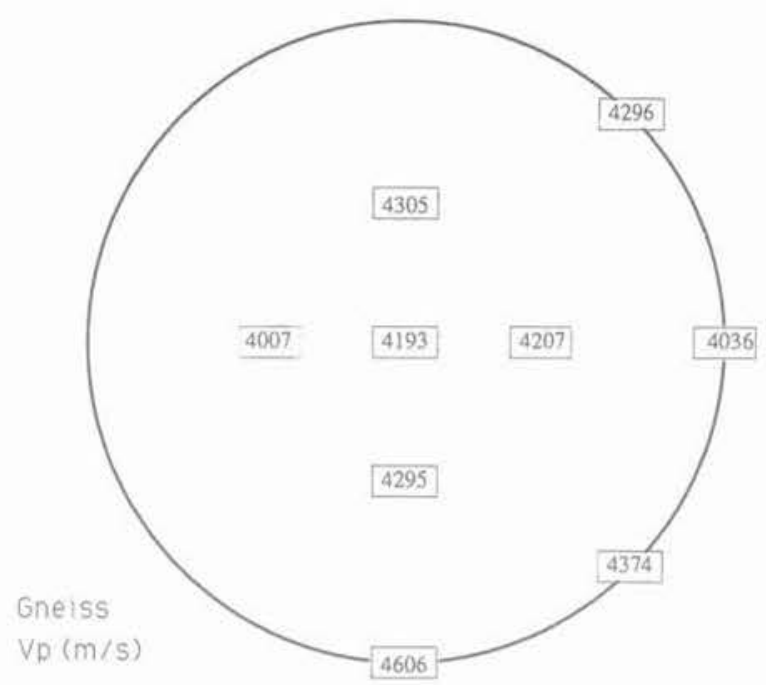

Fig. 2b. - Vitesses d'une onde $P$ dans un gneiss. Fig. $2 b$. - $P$ wave velocity in a gneiss.

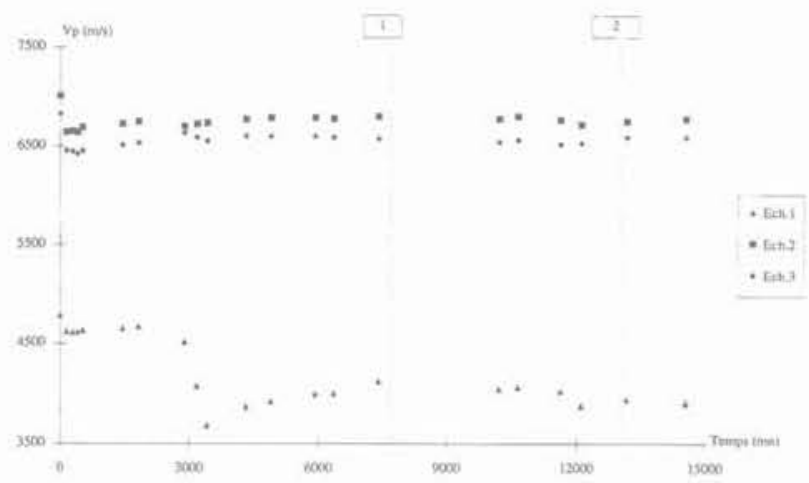

Fig. 3. - Evolution de la vitesse avec la désaturation de l'échantillon (1-2: étuvage à $60^{\circ}$ puis à $105^{\circ} \mathrm{C}$ ).

Fig. 3. - Velocity variation versus sample desaturation.

tantes (environ $5 \%$ ). En effet, la porosité déterminée par prise d'eau par saturation sous vide varie selon les échantillons de 0,5 à $1,7 \%$. Un essai de détermination de la porosité par porosimétrie au mercure n'a pas donné de bons résultats $(n \approx 0,14 \%)$, l'accès aux * pores » étant de dimension trop réduite. Pour cette raison, l'anisotropie reste peu influencée par la présence de fluide.

Pour des raisons expérimentales ces échantillons ont été stockés dans l'eau avant de subir différents essais à l'état $\propto \sec »$. Nous avons alors utilisé les ultrasons afin de distinguer les éprouvettes sèches des éprouvettes encore partiellement saturées. Des mesures de célérité de l'onde P ont été effectuées dans le temps alors que l'échantillon séchait à l'air libre puis à l'étuve (fig. 3).

Au début, la variation de vitesse suit la diminution de saturation en eau de l'échantillon. Au bout d'un certain temps, alors que tout semble stabilisé, on peut observer une brusque chute de vitesse. Celle-ci ne s'observe que lorsque la propagation de londe se fait perpendiculairement au plan de schistosité. Ce phénomène se produisant environ au bout de 50 heures 
de séchage correspondrait à un "décollement » de certains plans de schistosité lorsque les forces capillaires sont annulées ou trop faibles. L'étuvage à $60^{\circ} \mathrm{C}$ puis $105^{\circ} \mathrm{C}$ ne provoque par la suite aucun changement notable.

Les mesures de célérité en immersion permettent, par comparaison avec les mesures à sec de connaître " l'état " de la roche. En effet, plus la différence de ces deux vitesses sera élevée plus la proportion de vides dans l'échantillon sera importante ; ceux-ci pourront être des fissures.

Il faut cependant prendre garde aux effets induits par le couplage. En immersion il sera beaucoup plus facile à réaliser qu'à $\mathrm{sec}$.

Durant nos travaux nous n'avons effectué des mesures que sur échantillons * secs » ou « totalement saturés »; les guillemets utilisés trahissent l'incertitude dans laquelle nous nous trouvons de vérifier l'état de saturation avec exactitude. De même, des mesures à saturation intermédiaire n'ont pas été réalisées, car il ne nous est pas possible de contrôler précisément la quantité et la distribution de fluide dans la roche. Donnons toutefois l'exemple du grès de Massillon, cité par Bourbier et al. (1986) : la vitesse à saturation partielle est inférieure à la vitesse à sec elle-même inférieure à la vitesse à saturation totale. Cela serait dû à une interaction des deux effets cités plus haut, à savoir l'effet de densité et l'effet de compressibilité.

\subsection{Influence de la contrainte}

Les variations de vitesse en fonction de la contrainte dépendent du type d'onde considéré et de l'orientation du plan d'anisotropie.

Ainsi, dans le cas des éprouvettes à schistosité transversale (notées T) la vitesse augmente progressivement puis se stabilise, cette stabilisation se faisant à partir d'un seuil de 15 à $20 \mathrm{MPa}$ coïncide avec la fermeture des fissures. Dans le cas d'éprouvettes à schistosité axiale (notées A) on n'observe pas une telle évolution, puisque les plans de fissuration se trouvent parallèles à laxe de la contrainte principale et à la direction de propagation des ultrasons.

La figure 4 fait apparaitre une augmentation de $V s$ plus importante que celle de $\mathrm{Vp}$ lorsque la direction

DV\%

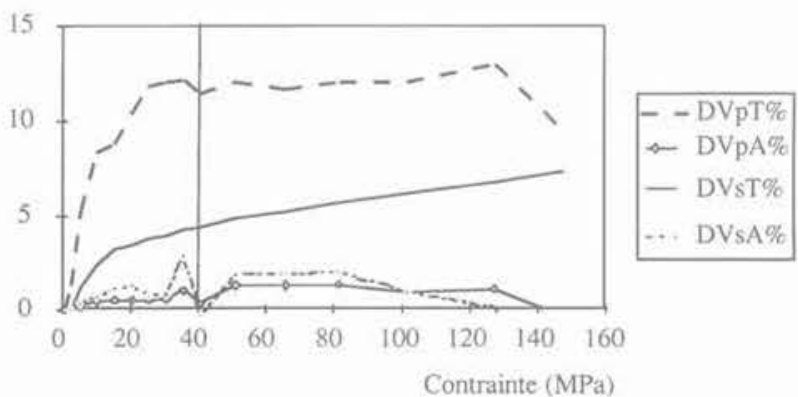

Fig. 4. - Variations de vitesse en fonction de l'état de contrainte

( $T$ : schistosité transversale: A: schistosité axiale).

Fig. 4. - Velocity variation versus state of stress

( $T$ : transversaly schistosity : A : axialy schistosity). de propagation (et donc d'application de $\sigma_{1}$ ) est orthogonale au plan d'anisotropie; cependant elle se stabilise rapidement alors que $\mathrm{Vp}$ continue d'augmenter lentement.

La représentation graphique adoptée nécessite quelques explications. L'essai est réalisé en deux étapes ; d'abord on augmente les contraintes de façon à garder un état de contrainte isotrope. Une fois le confinement désiré atteint, on augmente le déviateur. Ainsi, sur tous les graphiques donnant l'évolution d'un paramètre en fonction de la contrainte, ces deux phases sont séparées par un trait plein, matérialisant la valeur du confinement.

Les échantillons $\mathrm{A}$ ne présentent pratiquement pas de variations de vitesse, la légère augmentation étant probablement à mettre sur le compte du couplage.

En ce qui concerne les valeurs d'anisotropie, on se rend bien compte qu'elles ne sont pas accessibles puisque au cours de l'essai nous n'avons qu'une seule direction de mesure. $\mathrm{Si}$, toutefois, nous comparons les valeurs obtenues sur des échantillons d'orientation différente, il apparaît (fig. 5) que l'anisotropie de Vp diminue lentement durant tout l'essai alors que l'anisotropie de Vs chute aux faibles valeurs de contrainte, puis évolue comme le rapport des vitesses longitudinales.

En règle générale, on observe une augmentation des vitesses avec la contrainte du fait de la fermeture de fissures. Ainsi pour les roches anisotropes traitées, cet accroissement a lieu essentiellement lorsque londe se propage perpendiculairement au plan d'anisotropie correspondant plus au moins à un plan de fissuration.

La diminution de vitesse pouvant être liée à un endommagement de la roche, n'a pas été détectée, puisque nous nous sommes limités, dans la plupart des cas, à la phase élastique. De plus, notre montage expérimental ne permet pas d'étudier des signaux s'étant propagés parallèlement à la direction d'application de la contrainte. Cependant, quelques essais, menés jusqu'à la ruine de l'échantillon, n'ont pas montré une telle variation, la rupture se faisant de façon trop rapide.

An V\%

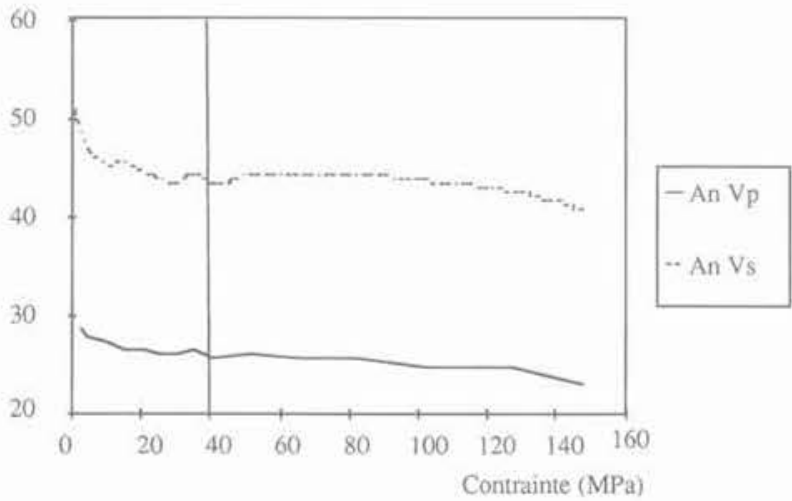

Fig. 5. - Evolution de l'anisotropie des vitesses en fonction de la contrainte.

Fig. 5. - Velocity anisotropy variation versus stress. 


\section{DÉTERMINATION DES MODULES ÉLASTIQUES}

Les vitesses des ondes dans une roche sont fonction de ses paramètres élastiques. La détermination de ceux-ci par une méthode ultrasonique présente des intérêts évidents :

- cette méthode n'est pas destructive ;

- elle nécessite un appareillage léger facilement transportable ;

- les résultats sont quasi-immédiats.

Nous allons écrire la loi de HOOKE dans un milieu quelconque :

$$
\sigma_{\mathrm{ij}}=\mathrm{C}_{\mathrm{ijkl}} \epsilon_{\mathrm{kl}}
$$

$\mathrm{C}_{\mathrm{ijkl}}$ représente les raideurs élastiques. Ces éléments verifient les relations :

$$
C_{i j k l}=C_{j i k l}=C_{k l i j}=C_{i j k}
$$

Ainsi, dans le cas le plus général, seulement 21 des 81 composantes du tenseur $C_{i j k l}$ sont indépendantes.

En combinant les équations (6), (1) et (3) on peut écrire l'équation du mouvement sous la forme :

$$
\left[\rho \frac{\partial^{2} u_{i}}{\partial t^{2}}=C_{i j k l} \frac{\partial^{2} u_{1}}{\partial x_{j} \partial x_{k}}\right]
$$

Une solution de cette équation peut s'écrire sous la forme :

$$
u_{i}=\Delta_{i} F\left(t-\frac{n j x_{j}}{V}\right)
$$
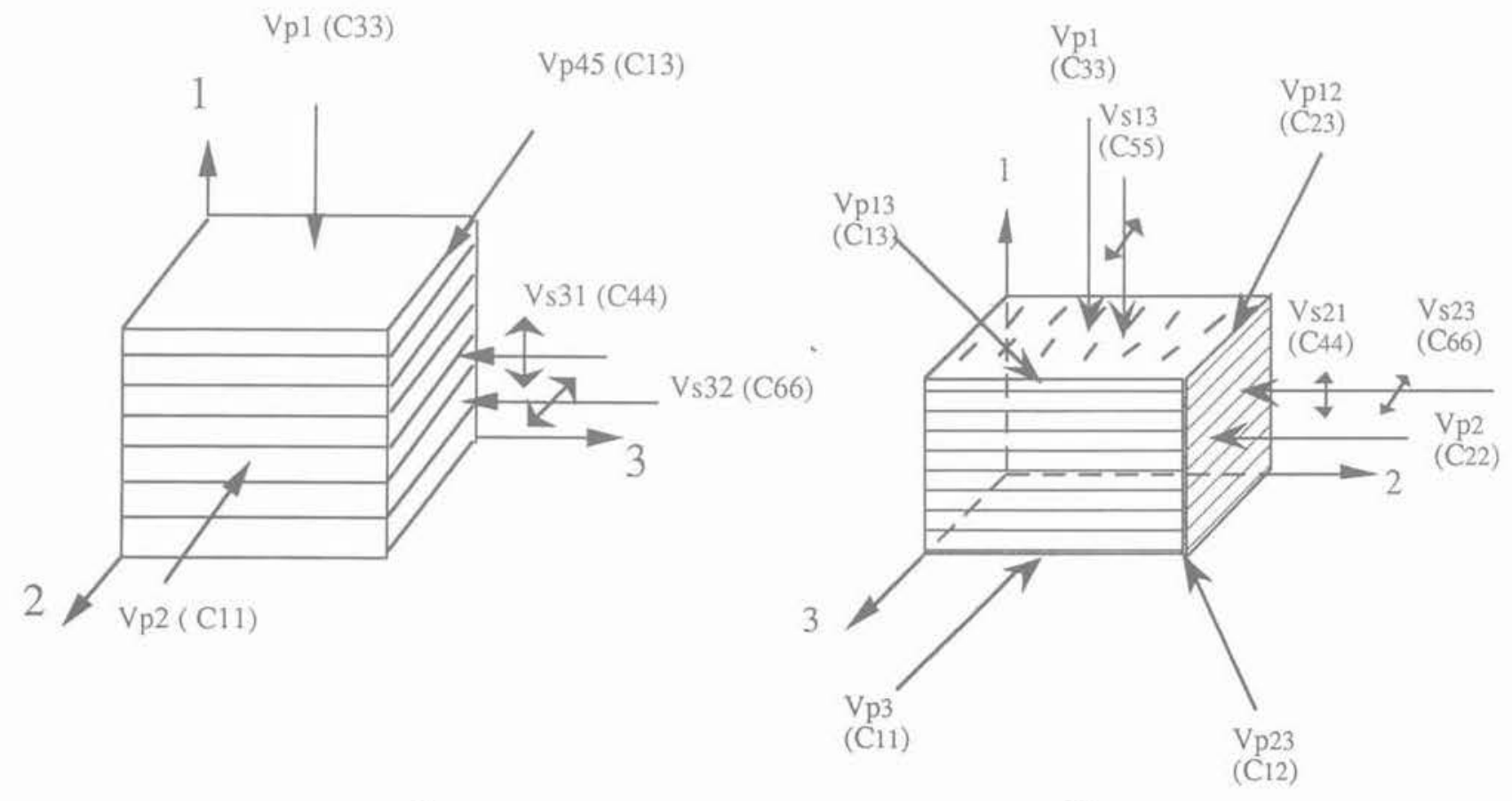

Reportant (8) dans (7): $C_{i j k l} n_{k} n_{j} \Delta_{j}-\rho V^{2} \Delta_{i}=0$. Posons $\Gamma \mathrm{ij}=C_{i j k l} n_{k} n_{j}$, il vient alors: $\Gamma_{i j} \Delta_{j}-\rho$ $\mathrm{V}^{2} \Delta_{\mathrm{f}}=0$ équation de CHRISTOFFEL.

- Tij est appelé tenseur de CHRISTOFFEL ou tenseur acoustique, celui-ci est symétrique :

- $n_{k} n_{j}$ sont les composantes d'un vecteur unitaire $\mathrm{n}$ définissant la direction de propagation de l'onde;

$-\Delta_{\mathrm{i}}$ représente la polarisation de l'onde.

On montre que $\Delta_{i}$ est vecteur propre du tenseur $\Gamma_{i f}$ et que celui-ci admet trois valeurs propres $\rho V_{1}^{2}$ vérifiant: $\operatorname{det}\left(\Gamma \mathrm{ij}-\rho \mathrm{V}_{1}^{2} \delta_{\mathrm{ij}}\right)=0$.

Donc, en un point d'un front d'onde existent trois directions de polarisation orthogonales, chacune étant associée à une vitesse $V_{i}$.

Selon la symétrie du matériau étudié, on détermine les directions de propagation et de polarisation nécessaires à la détermination de tous les paramètres élastiques. Les modules ainsi calculés sont dits dynamilors d'essais en compression.

Les figures $6 \mathrm{a}$ et $6 \mathrm{~b}$ montrent les directions de propagation et de polarisation minimales pour calculer la matrice de raideur. Entre parenthèses figurent les paramètres $C_{i j}$ obtenus à partir des mesures; à quelques exceptions près, ces valeurs ne sont pas directement accessibles par une seule mesure de vitesse.

Les tableaux I, II et III donnent les modules calculés pour le gneiss, le schiste et pour une argilite. Afin de simplifier les calculs et étant donné la faible valeur de Panisotropie linéaire, le schiste et l'argilite ont été traiques par opposition aux modules statiques obtenus

$$
6 \text { a }
$$

Vs32 (C66)

$6 \mathrm{~b}$

Fig. 6a et b. - Directions de propagation et de polarisation minimales nécessaires au calcul de la matrice de raideur d'une roche isotrope transverse (a) et orthotrope (b). Fig. $6 a$ et $b$. - Propagation and polarization directions necessarly to calcul elasticity tensor of a transversaly isotropic rock (a) and orthotropic rock (b). 
Tableau I. - Modules dynamiques du gneiss. Table 1. - Dynamics modulus of gneiss.

\begin{tabular}{|l|l|l|}
\hline$E_{1}=43740 \mathrm{MPa}$ & $G_{12}=19285 \mathrm{MPa}$ & $V_{12}=0,20$ \\
\hline$E_{2}=43470 \mathrm{MPa}$ & $G_{13}=21620 \mathrm{MPa}$ & $V_{13}=0,14$ \\
\hline$E_{3}=58350 \mathrm{MPa}$ & $G_{23}=22350 \mathrm{MPa}$ & $V_{23}=0,13$ \\
\hline
\end{tabular}

Tableau II. - Modules dynamiques du schiste. Table II. - Dynamics modulus of slate.

\begin{tabular}{|l|l|l|}
\hline$E_{1}=58870 \mathrm{MPa}$ & $G_{21}=15080 \mathrm{MPa}$ & $V_{12}=0,11$ \\
\hline$E_{2}=121300 \mathrm{MPa}$ & & $V_{23}=0,15$ \\
\hline
\end{tabular}

Tableau III. - Modules dynamiques de l'argilite. Table III. - Dynamics modulus of shale.

\begin{tabular}{|l|l|l|}
\hline$E_{1}=7885 \mathrm{MPa}$ & $\mathrm{G}_{21}=5995 \mathrm{MPa}$ & $V_{12}=0,05$ \\
\hline$E_{2}=29290 \mathrm{MPa}$ & & $V_{23}=0,08$ \\
\hline
\end{tabular}

tés en symétrie hexagonale. Les différentes valeurs montrent bien l'importance des anisotropies (les indices des modules se réfèrent aux axes de la figure 1).

Le tableau IV donne les coefficients de variation calculés sur les modules obtenus par ultrasons et par essais de compression sur le schiste. On constate que les modules dynamiques sont donnés avec un minimum de dispersion.

Tableau IV. - Disperson des mesures dynamiques

Table IV. - Dispersion of dynamics and statics measures

\begin{tabular}{|c|c|c|c|c|c|}
\hline & $E_{1}$ & $E_{2}$ & $V_{12}$ & $V_{23}$ & $V_{21}$ \\
\hline $\begin{array}{c}C V(\%) \\
\text { Dyn }\end{array}$ & 4 & 1,3 & 2 & 6,6 & 3,4 \\
\hline $\begin{array}{c}\text { CV (\%) } \\
\text { Stat. }\end{array}$ & 17 & 12 & 27 & 17 & 30 \\
\hline
\end{tabular}

\section{LA BIRÉFRINGENCE DES ONDES S}

Terminons ce bref aperçu avec les méthodes basées sur la biréfringence de l'onde S. Rappelons que ces ondes sont polarisées dans un plan, le déplacement des particules se faisant perpendiculairement à la direction de propagation. Cela implique que, pour obtenir un signal optimum, les plans de polarisation des transducteurs émetteurs et récepteurs soient confondus. Considérons un matériau homogène et isotrope ; si nous faisons tourner le plan de polarisation d'une céramique piézoélectrique d'un angle $\beta$, le signal reçu va diminuer d'amplitude et s'annuler pour $\beta=90^{\circ} \pm 180^{\circ}$. Il sera nul pour $\beta=180^{\circ} \pm$ $360^{\circ}$.
Considérons maintenant un matériau contenant un plan d'anisotropie parallèle à la direction de propagation de l'onde. Lorsque plan d'anisotropie et plan de polarisation seront confondus, l'amplitude du signal et sa vitesse seront maximales, inversement lorsque les deux plans seront orthogonaux.

Pour une position intermédiaire, le signal va se décomposer en deux composantes, lune polarisée selon le plan d'anisotropie, l'autre étant perpendiculaire. Ainsi, à la réception on aura deux signaux dont l'un sera d'amplitude et de vitesse supérieure à lautre.

On peut également faire tourner le plan de polarisation d'un transducteur, l'éprouvette étant fixe. Si la roche présente une anisotropie, le signal va s'annuler pour un angle différent de $90^{\circ}$.

Ce phénomène, appelé biréfringence, peut permettre la détermination d'anisotropies et leur orientation. La figure 7 schématise ce phénomène.

On peut également, grâce à ce phénomène, accéder à un grand nombre de vitesses sur un même échantillon et remonter ainsi à la matrice de raideur.

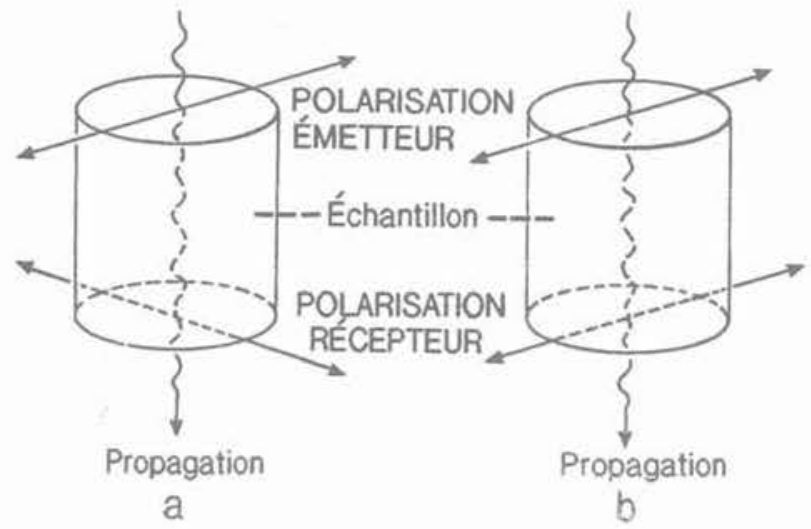

Fig. 7. - Principe de la biréfringence de l'onde $S$ (d'après RASOLOFOSAON P.N.J. et al. 1989).

a : Polarités perpendiculaires; $b$ : Polarités parallèles. Fig. 7. - S wave scattering principle.

a: perpendicular polarity; $b$; parallel polarity.

\section{CONCLUSION}

Les mesures par propagation d'ondes ultrasoniques sont faciles à mettre en œuvre et donnent très rapidement des résultats avec une précision remarquable. Elles sont bien adaptées à la détermination d'anisotropies. La matrice de raideur peut être calculée de façon relativement simple. Certains paramètres élastiques, difficilement déterminables à l'aide d'essais de compression, sont obtenus facilement (module de cisaillement).

L'étude de la biréfringence de l'onde de cisaillement peut apporter davantage de renseignements sur l'anisotropie.

Il faut, toutefois, prendre garde lors des mesures et lors de l'interprétation; ce genre de mesure demande un soin extrême durant l'expérimentation. 


\section{BIBLIOGRAPHIE}

ATTEWEL P.B. (1970), Triaxial anisotropy of wave velocity and elastic moduli in slate and their concordance with fabric and tectonic symmetry. Int. J. Rock. Mech. Min. Sci. and Geomech. Abstr., vol. 7, pp. 193-207.

CRAMPINS S. (1985), Evaluation of anisotropy by shear wave splitting. Geophysics, vol. $50, n^{\circ} 1$, pp. 142-152.

CUXAC P. (1991), Propagation et atténuation des ondes ultrasoniques dans des roches fissurées et anisotropes. Thèse INPL-ENSG.

CUXAC P., HOMAND F., HAMMADE E. et MOREL E. (1990), Comportement mécanique d'un schiste ardoisier. CR $25^{\circ}$ colloque GRF et AUGC, Grenoble, pp. 101 et 112 .
DIEULESAINT E. et ROYER D. (1974), Ondes élas. tiques dans les solides. Application au traitement du signal. Masson Editeur, Paris, $407 \mathrm{p}$.

GUYADER J. et DENIS A. (1986), Propagation des ondes dans les roches anisotropes sous contrainte. Evaluation de la qualité des schistes ardoisiers. Bulletin AIGI, n 33 , pp. 49-55.

LUCET N. et ZINSZNER B. (1989), Effet de la pression de confinement sur l'atténuation intrinsèque des ondes de fréquences soniques. Méthodologie de laboratoire et résultats pour les roches non argileuses. In Rock at Great Depth. CR Symp. Internat. Mec. Roches, Pau, pp. 257-264.

RASOLOFOSAON P.N.J. et ZINSZER B. (1989), Effet de la relaxation des contraintes in situ sur lanisotropie ultrasonore $P$ et $S$ des carottes de forage. In Rock at Great Depth. CR Symp. Internat. Mec. Roches, Pau, pp. 305-312. 